\title{
Article
}

\section{Searches for Exotic Interactions Using Neutrons}

\author{
William Michael Snow ${ }^{1, *(\mathbb{D}}$, Chris Haddock $^{2}$ and Ben Heacock ${ }^{2}$ \\ 1 Department of Physics, Indiana University, 727 E. Third St., Bloomington, IN 47405, USA \\ 2 National Institute of Standards and Technology, 100 Bureau Dr., Gaithersburg, MD 20899, USA; \\ christopher.haddock@nist.gov (C.H.); benjamin.heacock@nist.gov (B.H.) \\ * Correspondence: wsnow@indiana.edu
}

check for updates

Citation: Snow, W.M.; Haddock, C.; Heacock, B. Searches for Exotic Interactions Using Neutrons. Symmetry 2022, 14, 10. https:// doi.org/10.3390/sym14010010

Academic Editors: Yuri A.

Kamyshkov, Zurab Berezhiani and Rabindra Mohapatra

Received: 31 October 2021 Accepted: 14 December 2021 Published: 22 December 2021

Publisher's Note: MDPI stays neutral with regard to jurisdictional claims in published maps and institutional affiliations.

Copyright: (c) 2021 by the authors. Licensee MDPI, Basel, Switzerland. This article is an open access article distributed under the terms and conditions of the Creative Commons Attribution (CC BY) license (https:// creativecommons.org/licenses/by/ $4.0 /)$.

\begin{abstract}
Slow neutrons possess several advantageous properties which make them useful probes for a variety of exotic interactions, including some that can form at least some components of the dark matter of interest for this issue of Symmetry. We discuss the relevant neutron properties, describe some of the recent work that has been done along these lines using neutron experiments mainly with cold and ultra-cold neutrons, and outline some interesting and exciting opportunities which can be pursued using resonant epithermal neutron interactions in heavy nuclei.
\end{abstract}

Keywords: dark matter; neutron physics; fifth forces; time reversal violation; time variation of fundamental constants

\section{Introduction}

By now, there is certainly no need to present a detailed argument for the scientific importance of the search for whatever "dark matter" is. Any physical mechanism which gives all indications that it is weakly interacting with normal matter, possesses about four times the total energy density of normal matter in the universe, and makes a major impact on the dynamics of galaxies to the point of domination, must be investigated from all possible angles. Even if one restricts themselves to the assumption that dark matter is a new particle or coherent field which interacts very weakly with normal matter, but through the usual mechanism of boson exchange and couplings consistent with quantum mechanics and relativity, one is still left to consider an enormous range of possible coupling strengths and exchange boson masses with values not far below the Planck scale to ultralight masses with Compton wavelengths that are the size of galaxies. One can try to constrain the possibilities somewhat by making sure that this new dark-matter particle does not ruin well-established successes of present cosmology, such as Big Bang nucleosynthesis, or of the theory of stellar structure and energy loss that much of astronomy is based on. This still leaves wide gaps in mass and coupling space for experimental laboratory investigation, and even in parameter regimes which astrophysical and cosmological arguments seem to disfavor, an upper bound from a laboratory experiment is certainly on surer ground and is still worth pursuing. Therefore, the intense effort to search for particle dark matter in many different regimes continues.

What do neutrons have to do with all of this? For many dark-matter researchers, they are one of the "nightmare" sources of background for a large number of experiments which have sought weakly-interacting particles (WIMPS). WIMP dark-matter experiments typically look for low-energy recoil events in a large mass detector medium located underground to suppress cosmic ray and other backgrounds. The "WIMP miracle" argument which so many researchers have found attractive argues that the abundance of a darkmatter particle with mass near the electroweak scale and weak couplings to ordinary matter can freeze out of thermal equilibrium in the early universe at the right value to match the energy density of dark matter today. If one assumes a WIMP mass near the electroweak scale and the $v \approx 10^{-3} \mathrm{c}$ relative velocity between the local collection of dark-matter particles and a detector on Earth, the resulting energy and momentum transfers to individual 
particles in many dark matter detector media are in ranges that can also be efficiently made by various sources of neutrons in the environment. The ability of the neutrons to penetrate large amounts of matter with no or minimal ionization also mimics WIMP characteristics. WIMP dark-matter detectors therefore must shield the active detector volume from neutron backgrounds as much as possible, and indeed, neutron beams are used to help calibrate the detector response to real WIMP events.

In this paper, we will review and discuss how neutron laboratory measurements can search for new interactions which could be (potentially part of) dark matter. Of course, there are many other reasons having nothing to do with dark matter to search for such interactions, and since all of these intellectual motivations are interesting in their own right, we will not pay too strict attention to whether or not a particular possibility really could contribute to the dark-matter issue or not. Rather, we will concentrate on why neutrons can carve out a broad niche for investigation of interesting possibilities for new weakly coupled interactions which are not so easy to access by other means. We will also make the case that there is much room for further experimental progress in this area, using both cold and ultra-cold neutron measurements which have dominated the experimental efforts so far, and also the so-called "epithermal" neutrons which are now available with high intensities in the new intense spallation neutron sources developed for neutron-scattering and nuclear data applications.

\section{Neutrons and Weakly-Coupled Interactions}

The electrical neutrality of the neutron, coupled with its small magnetic moment, small neutron-electron scattering amplitude (which means that neutron cross-sections are set by nuclear, rather than atomic distance scales), and very small electric polarizability frees it from the electromagnetic backgrounds faced by searches for exotic interactions which use test masses made of atoms. This is a very important feature of neutrons in light of the experimental strategy one would naturally employ to look for a new weaklycoupled interaction; namely, bringing two large tests masses close together and trying to measure a feeble force. This strategy works well until one considers force ranges which are smaller and smaller. In this case, the more or less constant density of the condensed matter that the test masses are made of starts to severely limit the amount of matter that can be brought together closely enough to see new interactions of shorter range. Additionally, one starts to run into strong electromagnetic backgrounds like the Casimir effect which is present for any matter made of atoms. Although the density of free neutrons in beams accessible for experimental work are far lower than condensed-matter densities, the neutron interactions with matter possess electromagnetic backgrounds which are also several orders of magnitude lower. One therefore expects that neutrons can be the best choice to search for new interactions with nucleons of sufficiently short range.

The related ability of slow neutrons to penetrate macroscopic amounts of matter due to the resulting small cross-sections and to interact in the medium with negligible decoherence also allows the quantum amplitudes governing their motion to accumulate large phase shifts, which can be sensed with various types of interferometric measurement [1-4]. These experiments are performed in an energy regime where only s-wave scattering is relevant. Specifically, when $k R \ll 1$ is satisfied, where $k$ is the neutron wave vector and $R$ is the range of the complicated and strong neutron-nucleus interaction, the scattering process can be described using only the target's scattering length density. Because scattering lengths have been experimentally and accurately measured for essentially all stable isotopes, one can cleanly interpret and analyze searches for the small, exotic effects of interest. Additionally, since there are several sources of intense neutron beams and sources with energies low enough to meet this $k R \ll 1$ condition which are made primarily for neutron-scattering studies in materials science $\left(k R=10^{-5}\right.$ for thermal neutrons), several different types of opportunities to conduct creative experiments exist.

These features of slow neutron interactions have therefore been exploited in several searches for possible new weakly coupled interactions of various types, including 
chameleon dark-energy fields, light $Z^{\prime}$ bosons, in-matter gravitational torsion and nonmetricity of spacetime, axion-like particles, and exotic parity-odd interactions [5-17]. A thorough review of almost all of the recent results from neutron measurements of this type has recently appeared [18], and we refer the reader to this well-written recent paper for more details on many of the experiments, as well as their interpretation. Another recent review [19] has placed this work in the context of analogous investigations using atomic measurements. We will be discussing a couple of characteristic examples of this work.

Before doing so, we briefly summarize some of the other intellectual motivations which have informed this work, much of which was not initially connected to the darkmatter problem. The possible existence of new interactions in nature with sub-millimeter ranges, corresponding to exchange boson masses above $1 \mathrm{meV}$ and with very weak couplings to matter has been discussed for some time $[20,21]$. Particles which might mediate such interactions started to be generically referred to as Weakly-Interacting sub-eV Particles (WISPs) about a decade ago [22]. The extended symmetries present in many theories beyond the Standard Model, including string theories, are typically broken at some high-energy scale, leading to weakly-coupled light particles with relatively longrange interactions, such as axions, arions, familons, and Majorons [23,24]. A continuous symmetry spontaneously broken at some scale $M$ generates a massless pseudoscalar mode which couples to massive fermions, $m$, with a coupling of order of $g=m / M$. When the symmetry is also explicitly broken at scale $\Lambda$, the mode can become a pseudo-Goldstone boson of order $m_{\text {boson }}=\Lambda^{2} / M$ [25]. Beyond the Standard Model, gauge theories can predict axial-vector bosons, such as paraphotons [26] and extra Z bosons [27] through this mechanism. Of course, new weakly-coupled interactions can possess some of the attributes of dark matter, so there is a lot of natural overlap between these two research areas.

One can conduct a reasonably general classification of interactions between nonrelativistic spin $1 / 2$ fermions assuming the usual constraints from relativity and quantum mechanics, which for the weakly-coupled interactions of interest leads to the usual exchange boson interaction mechanism, supplemented with (in general, spin-dependent) couplings at the vertices. In one heavily-used parametrization [28], neglect of higher-order relativistic terms, which was justified by the intended application of this work to laboratory experiments involving at least one macroscopic test body, left only 16 linearly independent nonrelativistic potentials composed from scalar invariants involving the spins, momenta, interaction range, and possible particle couplings. Of these sixteen interactions, which came from the single exchange of a spin 0 or spin 1 boson, one was spin-independent (the simple Yukawa potential), six depended on the spin of one of the particles, and the remaining nine depended on both particle spins. Ten of these sixteen possible potentials depended on the relative momenta of the particles. Later work [29] reformulated the mixed position/momentum representation in which the initial results of Dobrescu and Mocioiu were expressed into the position representation, which is especially relevant for application to the analysis of nonrelativistic bound systems, like atoms.

The well-timed paper of Dobrescu and Mocioiu inspired a very wide variety of experimental work from researchers in neutron, atomic, gravitational, and condensed matter physics who recognized the great untapped potential for sensitive experimental searches for these different interactions with sub-millimeter ranges. This work continues to be unabated to this day. There had already been decades of work looking for new forces with millimeter and longer ranges in gravitational physics, and the fantastic precision possible in atomic spectroscopy is well-known, but almost none of the intermediate length scales in between had ever been the subject of serious experimental study for possible new interactions of nature. Likewise, there are fewer experimental searches for weakly-coupled interactions over distance scales between atomic and nuclear length scales. Furthermore, most (but not all) experiments involving atomic structure naturally address exotic interactions of electrons, so if the new weakly coupled interaction involves only nucleons, there are even fewer experimental constraints. In particular, the addition of the spin degree of freedom opens up a variety of possible new interactions to search for, which might have 
escaped detection to date. Powerful astrophysical constraints on exotic spin-dependent interactions [30-32] have been made using stellar energy-loss arguments, sometimes in combination with laboratory tests of spin-independent interactions [33]. Nevertheless, it was thought by most experimental researchers that a laboratory experiment is well-worth doing, even if it might be disfavored by a cosmological or astrophysical argument, in view of the possibility that some other piece of physics might invalidate an indirect argument. For example, the spin-independent exotic chameleon mechanism could, in principle, invalidate some of the astrophysical bounds, while having a negligible effect in cooler, less dense lab environments [34], and the astrophysical bounds from energy balance arguments in stellar and supernova analyses were not very stringent for axial-vector interactions [28]. The wide range of theories which produce exotic spin-dependent interactions which can escape astrophysical constraints with these and other loopholes, along with the intrinsic value of controlled laboratory experiments, have led to more and more searches for exotic spin-dependent interactions. Recent work [35] has placed this effort in a broader theoretical context.

The rapid progress which has been made in the searches conducted to date is in part due to the preexistence of other types of experimental work which could be repurposed for this type of investigation. Examples include the experimental apparatus used to test the gravitational equivalence principle, experiments used to study the Casimir effect, development of laser control of nanoparticles, and advances in micromachined high-Q mechanical oscillators. For possible exotic spin-dependent interactions, the sensitive types of spin-dependent measurement techniques employing light and magnetic fields developed in AMO physics involving electron and nuclear spins for delicate NMR spectroscopy and in searches for electric dipole moments of atoms and molecules could be easily adapted to exotic spin-dependent interaction searches with minimal changes in existing setups, or even with reanalysis of existing data taken for other purposes. Many works of this type quickly ruled out huge swaths of hitherto unexplored parameter regimes in the log-log space of exchange boson masses and coupling constant products.

Later in this work, an interesting overlap with a set of dark matter models was developed, which could also be analyzed in a reasonably general way using the techniques of the effective field theory. Fichet [36] observed that certain candidates for dark matter in the sub-GeV mass range can induce Casimir-Polder-type interactions between nucleons which can be sought using experiments sensitive to force ranges from nuclear to atomic scales. Researchers [37] quickly used some of the experimental work conducted to search for the various weakly coupled interactions mentioned above and applied it to this task. Again, it was found that, over a broad range of mass scales for dark-matter particles which can include such nucleon interactions, neutron measurements formed some of the most restrictive constraints.

Of the broad experimental activity devoted to this research area, neutron measurements have been most competitive compared to other systems in three general areas: (1) searches for possible exotic Yukawa interactions coupled to matter with ranges below those accessible to Casimir force measurement methods, (2) searches for exotic gravitational effects of various types, including searches for chameleon and symmetron dark energy fields, and (3) searches for spin- and velocity-dependent interactions from the spin- 1 boson exchange, which can be generated by a light vector boson $X_{\mu}$ coupling to a fermion $\psi$ with an interaction of the form $\mathcal{L}_{I}=\bar{\psi}\left(g_{V} \gamma^{\mu}+g_{A} \gamma^{\mu} \gamma_{5}\right) \psi X_{\mu}$, where $g_{V}$ and $g_{A}$ are the vector and axial couplings. For this last example in the nonrelativistic limit, this interaction gives rise to two interaction potentials of interest depending on both the spin and the relative momentum [9]: one proportional to $g_{A}^{2} \vec{\sigma} \cdot(\vec{v} \times \hat{r})$, and another proportional to $g_{V} g_{A} \vec{\sigma} \cdot \vec{v}$. In all three of these cases, experimental systems could be identified which allowed the advantages of neutrons discussed above to be realized through one type of neutron interferometric measurement, or another which involved some coherent interaction of the neutron with a macroscopic system. 
Here, we present neutron searches for exotic gravity as an example. Many theories beyond the Standard Model postulate short-range modifications to the $1 / r$-dependence of the Newtonian gravitational potential, such as the existence of compact extra dimensions of spacetime accessible only to the gravitational field [38-40] or the $\sim 100 \mu \mathrm{m}$ length scale of the density of dark energy [41]. A common experimental parameterization of modified gravity is the Yukawa potential $V^{\prime}(r)=-\frac{G M m}{r}[1+\alpha \exp (-r / \lambda)]$ [42], for which neutron-scattering currently gives the best constraints on $\alpha$ for $\lambda$ between $10^{-8} \mathrm{~m}$ and $10^{-13} \mathrm{~m}$. Constraints have been made by analyzing the dependence of neutron-nucleus scattering lengths on neutron energy and target mass number [43], the angular distribution of neutrons scattered from noble gases [44,45], the energy dependence of neutron-nucleus cross-sections in lead [5,46], and very high-energy forward cross-section measurements at accelerator facilities [47].

One example of an experimental search for deviations from the inverse square law of gravity at the nanometer-length scale uses slow neutron-scattering from noble gases, typically at around atmospheric pressures. By measuring the neutron momentum transfer dependence of the differential cross-sections of the noble gases and comparing to their wellknown analytical forms, upper bounds may be placed on the strength of a new interaction as a function of interaction length $\lambda$, which are particularly sensitive in the region $\lambda \leq 1 \mathrm{~nm}$.

A neutron-xenon scattering measurement performed by Kamiya et al. at the HANARO research reactor of the Korean Atomic Energy Research Institute [44] was used to constrain $\alpha$ over the range of $\lambda=0.01 \mathrm{~nm}$ to $\lambda=10 \mathrm{~nm}$. Following that measurement, a study of neutron-helium and neutron-xenon scattering performed on the low-divergence beam branch of the Neutron Optics and Physics (NOP) beam line at J-PARC was published in 2018 [45], with improved results in the low $\lambda$ region due to the higher momentum transfer acceptance of the scattering apparatus (see Figure 1). Since then, the J-PARC group has moved their gas handling set-up to the NOVA beam line (BL-21) at J-PARC, which has a very large momentum transfer acceptance rate $\left(0.3 \mathrm{~nm}^{-1}\right.$ to $\left.1000 \mathrm{~nm}^{-1}\right)$, and has since taken several days' worth of neutron-scattering data on xenon, krypton, and neon gas samples at higher pressures ( $\sim 7 \mathrm{bar}$ ) with the goal of increasing the statistical precision by at least an order of magnitude in the shorter $\lambda$ region. Data taken in 2021 are now under analysis.

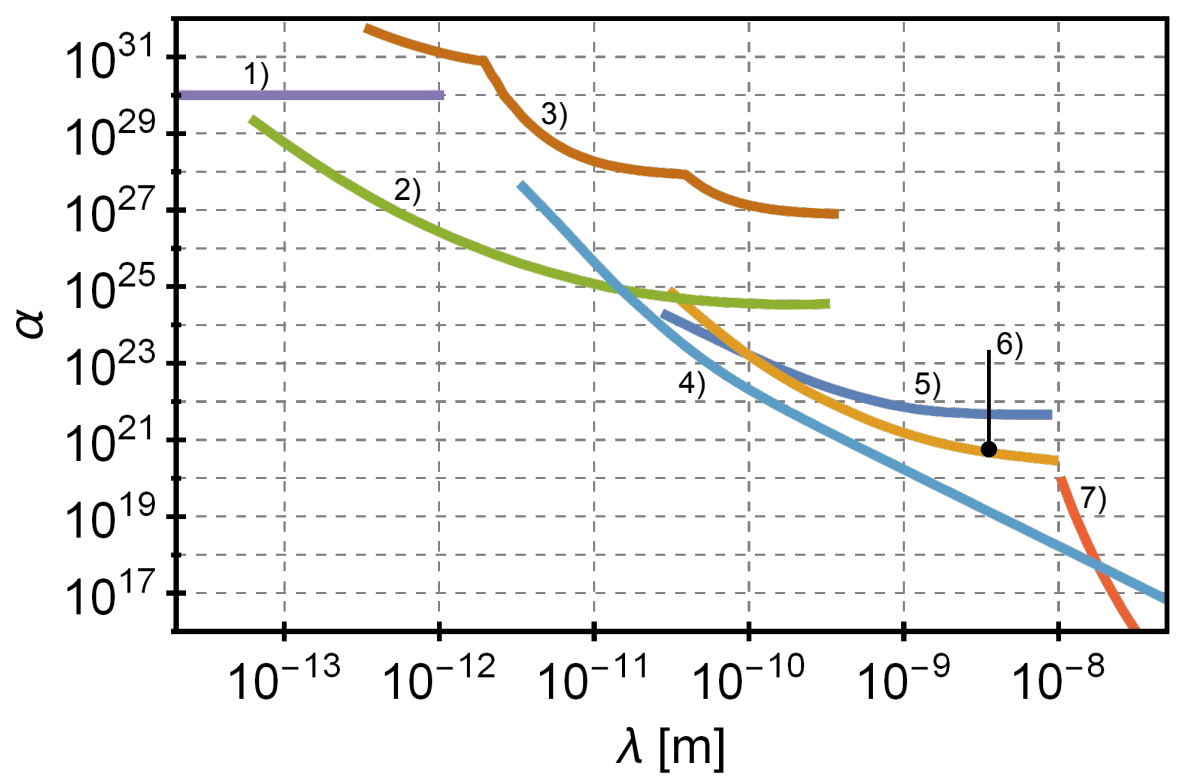

Figure 1. Limits on the strength and range of short-range gravitational interactions of matter using neutrons and other probes in the $10^{-8} \mathrm{~m}$ to $10^{-13} \mathrm{~m}$ range. Regions above the curves are excluded (95\% confidence). Limits include those from (1) the LHC [42], (2) neutron- ${ }^{208} \mathrm{~Pb}$ scattering [46], (3) exotic atom spectroscopy [48], (4) neutron pendellösung interferometry [49], (5,6) neutron-noble gas small-angle scattering [44,45], and (7) Casimir force [50]. 
The main limitations on the sensitivity of the neutron-noble gas-scattering measurements come from the relatively large size of the nuclear scattering background compared to scattering from the exotic potential (about 1000:1) and the small mass of the atoms. This can, in principle, be compensated for by using coherent scattering from larger mass objects, such as nanoparticles, as the exotic potential is proportional to the product of the neutron mass and the mass of the target-scattering center. In order to avoid a proportional increase in a nuclear scattering background from the nanoparticle, one can take advantage of the existence of stable nuclei with coherent scattering amplitudes of different signs and use a composite powder consisting of particles with different signs, combined in a ratio that satisfies $\sum_{i} p_{i} a_{i} \approx 0$, where $p_{i}$ and $a_{i}$ are the concentration and the coherent scattering length of the $i$-th element of the nanoparticle powder. The same group who is pursuing the neutron-noble gas-scattering studies at J-PARC has begun development of a nanopowder target containing a vanadium $(a<0)$ and nickel $(a>0)$ alloy supported by an ethylenemethyl-acrylate copolymer (EMA) film. The total neutron-scattering cross-section of the EMA film is over four orders of magnitude lower than the cross-section of the nanopowder target, and thus contributes negligibly to the background signal. The group has performed simulations to estimate the potential sensitivity for this measurement, and has found that slow neutron-scattering measurements from such a composite nanopowder target performed over a comparable data acquisition time as the noble gas-scattering studies can, in principle, reduce the upper bound on $\alpha$ from noble gas-scattering by at least two orders of magnitude over the range of $\lambda>1 \mathrm{~nm}$.

Another neutron method which can be employed to conduct sensitive searches for exotic interactions is neutron dynamical diffraction in large, perfect crystals. Recent measurements using pendellösung neutron interferometry have improved limits for Yukawamodified gravity between 20 pm and $10 \mathrm{~nm}$ [49], as is shown in Figure 1. Pendellösung interferometry measures the beating period between neutron wave states within a Braggdiffracting crystal, where wave function nodes occur either on or in-between the Bragg planes. The state with nodes on the Bragg planes experiences a reduced optical potential, whereas the state with nodes between the Bragg planes accrues a phase from the neutronatomic potential. For crystals of sufficient quality and very low strain, the two internal states interfere coherently, causing a spatial beating period between the diffracted and transmitted beams, modulated by tilting the crystal and changing the optical thickness. The absolute phase of the interference pattern provides a $\sim 5 \times 10^{-5}$ relative precision measure of the crystalline potential evaluated at a momentum transfer determined entirely by the Bragg plane spacing. In contrast to constraints on undiscovered spin-dependent interactions which employ diffraction from non-centrosymmetric crystals to prevent quadratic suppression of spin-dependent couplings [51], pendellösung interferometry is best used with higher-symmetry crystal structures, such as silicon's diamond unit cell. Fitting silicon structure factors from pendellösung interferometry only requires a single degree of freedom to describe solid state effects in the form of the thermal mean square displacement, whereas a crystal with less symmetry requires mean-square displacement matrices with up to six degrees of freedom for every member of the crystal's primitive cell. The simple functional dependence on thermal vibrations of some crystal structures for pendellösung interferometry is a unique strength compared to the static structure factor corrections needed for epithermal neutron total transmission measurements [52], which along with accounting for nuclear resonances is a possible culprit for tension in neutron charge radius values from different research groups [49]. With only three out of possibly tens of structure factors in silicon and only one structure factor measured in germanium, which also has a diamond structure, expanded pendellösung interferometry measurements in these two crystals are expected to improve Yukawa-modified gravity limits by at least a factor of five in the near future. This could be done with high efficiency while, simultaneously reducing systematic uncertainties by measuring a set of harmonic Bragg reflections on a pulsed beam [53]. More speculative experiments include performing pendellösung interferometry on a wider set of crystal types, as well as spin-resonant pendellösung, where a resonance effect occurs when 
the Larmor precession distance from a laboratory magnetic field matches the pendellösung length. Because both interactions have the same dependence on neutron energy, the resonance field depends only on the momentum transfer of the Bragg reflection; the resonant field for the (111) reflection is silicon is about $6 \mathrm{kG}$ [54]. Alternatively, tuning the Larmor precession distance to the width of a channel-cut multi-bounce crystal may be sensitive to exotic spin-dependent interactions, even in crystal species that are centrosymmetric [55].

The prospects for continued experimental progress are excellent. Ultra-cold neutrons are employed in gravity resonance spectroscopy [2,56], which creates coherent superpositions of bound states of neutrons formed in a potential from Earth's gravitational field and a flat mirror. One can drive and resolve resonance transitions using acoustic transducers in a vibrational version of Ramsey spectroscopy. qBOUNCE has successfully conducted proof-of-principle measurements demonstrating vibrational Rabi spectroscopy [57], and vibrational Ramsey spectroscopy [58], and has sought several different types of exotic interactions $[12,17,59-61]$ through the influence of interactions sourced by the mirror material on the neutrons [62]. The most recent example is a search for possible CPT/Lorentz violation in the interactions of neutrons with the gravitational field of the Earth [63]. The GRANIT UCN spectrometer [64] at the ILL/Grenoble can conduct precision measurements on UCN gravitational bound states [65] using magnetic field gradients [66] with higher statistics when it is fed by a superfluid-helium-based UCN source [67]. With a bright, very cold neutron (VCN) source, one could employ a Lloyd's mirror interferometer $[68,69]$ to look for exotic interaction phase shifts from the mirror surface.

\section{Future Opportunities using Epithermal Neutrons}

The great majority of the examples discussed so far have made use of cold and ultracold neutrons, and there are many experimental opportunities for continued progress using neutrons in this energy range, as most of these experiments are not yet limited solely by the statistical accuracy available in beams and sources at present neutron research facilities. Neutron-scattering from nanoparticles, further improvements on the recent results using neutron dynamical diffraction, and gravity resonance spectroscopy all hold promise for future improvements in sensitivity. There is also an abundance of new ideas for systems to study which can support delicate neutron interferometric measurements. However, all of the arguments given above for the value of neutrons in this type of research also apply to neutrons of significantly higher energies. We will follow the lingo of the field and call neutrons in this energy range "epithermal" neutrons. One of the new physical phenomena which appear in this energy regime are several sharp neutron-nucleus resonances, which are especially plentiful in heavy nuclei with their high-level densities near the neutron separation energy. The much longer time (factors up to $10^{6}$ ) that a neutron spends in the nucleus in a resonance reaction compared to a potential scattering reaction provides an opportunity to greatly amplify the small effects of the exotic weakly-coupled interactions of interest.

These neutrons, whose energies are in the $\mathrm{eV}$ range and above, are not produced with high intensities at the neutron research reactors where many of the measurements referred to above have been conducted. Intense sources of $\mathrm{eV}$ neutrons come in two main varieties: steady-state sources and pulsed sources. Hot moderators at reactor sources produce high eV neutron fluence, but only over a limited energy range. eV neutrons and above are produced over a much broader range of neutron energies at steady spallation neutron sources, such as PSI and at pulsed neutron sources, which enable neutron energy measurements using neutron time-of-flight. Pulsed eV neutrons have been produced using active motion of a split-fissile assembly at Dubna, $(\gamma, n)$ photoneutron production using bremsstrahlung induced in heavy metal targets with intense pulsed electron linacs, and now spallation neutron sources, such as LANSCE, SNS, JPARC [70], CSNS, ISIS, and the ESS driven by $\mathrm{GeV}$ energy proton accelerators with time-averaged powers now into the MW regime. These accelerator-based neutron sources have been developed both for neutron-scattering and materials science studies, and are also to help conduct the nuclear 
spectroscopy needed for nuclear structure and reactions, astrophysics, nuclear fission, and applied nuclear data measurements.

These new sources enable opportunities for experiments in the area of interest for this paper. With the addition of a polarized neutron beam capability, several new and exciting scientific investigations can be conducted with epithermal neutrons, including searches for spin-dependent exotic interactions. Polarized eV neutrons can be produced efficiently now over the $1 \mathrm{eV}$ to $10 \mathrm{eV}$ range using neutron spin filters based on spin-exchange optical pumping of ${ }^{3} \mathrm{He}$ [71] and over the $1 \mathrm{eV}$ to $100 \mathrm{keV}$ energy range with polarized proton targets, either produced in the well-developed traditional dynamically polarized proton targets [72] similar to the type used in nuclear and high-energy physics for spin-dependent strong interaction studies, or using the new laser-based polarized proton neutron spin filters $[73,74]$. Examples of new scientific investigations which could be performed with eV-keV polarized neutron beams include, but are not limited to, the following areas and topics in science and technology:

(1) Laboratory Cosmology: According to the Sakharov conditions, new sources of time-reversal violation are needed to explain the anomalously large observed baryon asymmetry of the Universe in Big Bang cosmology. Certain neutron-nucleus resonances can act as tremendous amplifiers of potential time-reversal-violating interactions, making the investigation of these nuclear systems an excellent candidate for uncovering new, Beyond the Standard Model (BSM) physics. Future activities in this program using a polarized eV beam can include continued spectroscopy work and the first-ever searches for time-reversal violation using polarized neutron transmission through polarized nuclear targets. The first such pioneering measurements will also place the most stringent constraints on axion-like particles (ALPs) over a range of ALP masses and couplings which are inaccessible by other methods. Measurements of forward-backward (longitudinal) and left-right (transverse) asymmetries in polarized eV-MeV neutron-scattering from nuclear resonances in heavy nuclei can also be used to search for both ALPs and other dark-matter candidates.

We discuss this subject in more detail below.

(2) Nuclear Statistical Spectroscopy: Parity-odd effects in neutron-nucleus resonances were discovered in 80 p-wave resonances in heavy nuclei by the TRIPLE collaboration at LANSCE in the 1990s $[75,76]$. Subsequent progress in the measurement of nucleon-nucleon parity violation in two- and few-nucleon systems [77,78], combined with progress in atomic physics enabling measurements of nuclear anapole moments [79], can soon be used to provide the input information needed to confront the predictions of nuclear statistical spectroscopy $[80,81]$ for parity-odd effects in neutron-nuclear resonances. Searches for parity violation in p-wave resonances in heavy nuclei not yet measured [82] and in the $(n, \gamma)$ cascades from p-wave neutron capture [83] can also provide important new information.

Polarized eV neutrons in combination with in-beam magnetic field wigglers (similar in concept to those used at synchrotron facilities) can be used to produce eV neutron beams with energy spreads about two orders of magnitude narrower than those achievable by neutron time-of-flight techniques. Such beams could be used to search for narrow p-wave resonances in nuclei, which are well-known to be strongly undersampled in current data sets. A sufficiently broad scan for $\mathrm{p}$-wave resonances in heavy nuclei can test the predictions of nuclear statistical spectroscopy.

(3) Neutron Quantum Optics and Entanglement: The recent demonstration at ISIS and ORNL of two- and three- variable single-neutron quantum entanglement in the spin, path, and energy qubits of polarized meV neutron beams [84,85] can be extended to the eV neutron energy range using neutron Wollaston prisms [86]. This development can open the new field of entangled neutron-scattering and enable neutron-nucleus resonance profile measurements of unprecedented precision. To our knowledge, a neutron-nucleus resonance has never been excited by an entangled neutron state. The physical phenomena which this work could access are completely unexplored. 
Neutron orbital angular momentum (OAM) states are of intellectual interest for possible applications in quantum sensing with neutrons and for neutron-scattering studies of materials, where the nonzero $\vec{L}$ of the beam can selectively couple to certain topological excitations in condensed matter. Neutron OAM was first demonstrated with slow neutrons using perfect crystal neutron interferometry [87] and then with magnetic quadrupole fields [88] and with orthogonal magnetic prisms [89], and it is clear that further improvement of the methods for neutron OAM generation and analysis are needed to realize a broader set of scientific applications $[90,91]$. Neutron OAM generation by strong nuclear and electromagnetic neutron spin-orbit interactions is also expected on theoretical grounds [92-94], and is now under active experimental investigation.

The tremendously large parity-odd asymmetry at the $0.7 \mathrm{eV}$-wave resonance in ${ }^{139} \mathrm{La}$ can also be used to impart orbital angular momentum to a transversely polarized $\mathrm{eV}$ neutron beam. Parity-odd neutron spin rotation [95] rotates the plane of polarization of a transversely polarized neutron beam about its momentum vector. By conservation of angular momentum, one expects that the neutron beam develops a nonzero $\vec{L}$ to conserve $\vec{J}$, as mentioned in the original paper describing this phenomenon [96], and explicit computations using simple models of the neutron-nucleus weak interaction potential confirm this expectation [97].

Demonstration of neutron OAM in the eV energy range would establish a new degree of freedom for $\mathrm{eV}$ neutron physics, which is also completely unexplored. Combined with other neutron optical devices, one can then create a neutron beam with single-particle quantum entanglement in up to four different dynamical variables in conditions which (as far as we know) cannot be achieved with photons or other particles.

(4) The Physics of Neutron-Induced Fission: The observation of very large parityodd effects in neutron-induced fission resonances [98-101] constitutes one of the clearest experimental demonstrations of the dominance of a relatively small set of quantum tunneling amplitudes near the fission barrier [102,103]. Extensive work led primarily by Soviet/Russian groups over several decades discovered many parity-odd and parity-even asymmetries in polarized neutron-induced fission resonances in several fissionable nuclei [104-106]. Later work in this field shifted mainly to measurement of formally T-odd correlations in fission product angular distributions to try to understand subtle features of fission dynamics by looking at various angular correlations in both binary and ternary fission [107-111].

However, all of this work was performed either with slow neutrons or with eV neutrons at low time-of-flight resolution pulsed neutron sources which could not resolve all the resonances of interest. Investigations of this phenomenon at short-pulsed spallation neutron sources with their much sharper neutron energy resolution can address several unanswered questions raised in this previous work. Measurements with polarized eV neutrons could clarify the physical mechanism behind (formally) time-reversal-odd effects observed in neutron-induced ternary fission, which are thought to be sensitive to the relative motion of the fission fragments upon scission. The time-reversal violation present in these fission processes is not fundamental in nature, but rather due to interference effects and final-state interactions of the reaction products emerging from the "cold" stage of fission. The size of these formally T-odd effects is therefore very sensitive to subtle dynamical features of fission that are inaccessible in other fission observables. If recent theoretical work [112] which suggests that one must consider interference between different resonance channels to understand these observations is correct, then one wants to measure with polarized $\mathrm{eV}$ neutrons from a short-pulsed spallation source to make further scientific progress.

With a polarized proton-based neutron spin filter which can efficiently polarize neutron beams over a very wide dynamic range from $\mathrm{meV}$ up to $100 \mathrm{keV}$, one could perform polarized neutron-induced studies of fission dynamics. Previous work has shown that neutron polarization-dependent asymmetries survive the fission process in several fissionable nuclei. Such measurements could supply completely new types of information on fission 
dynamics, which perhaps can confront the exciting recent developments in the quantitative understanding of fission.

We now elaborate on two example ideas which fall within this general set of intellectual opportunities. One effort now in preparation is a search for a neutron-polarized nuclear target interaction which is simultaneously odd in the parity and time-reversal quantum numbers using a polarized neutron optic methodology. Another more speculative idea is to use the rapid variation of the neutron-nucleus scattering amplitude near a sharp neutron-nucleus resonance to look for a time variation of the nucleon-nucleon interaction, similar to analogous searches in atomic physics which look for a time variation in the fine structure constant. In each of these cases, the advantage of neutrons is the possibility to exploit neutron-nucleus resonances along with the neutron spin degree of freedom as "amplifiers" for the tiny effects of interest.

\section{Time-Reversal Violation in Neutron Optics and a Search for Axion-like Particles}

In order to explain the baryon asymmetry of the universe in Big Bang cosmology according to the Sakharov argument [113], new sources of time-reversal violation are necessary. When neutrons interact with heavy nuclei, they may experience certain compound nuclear p-wave resonances. These resonances can be used to search for P- and T-odd interactions via a term in the neutron forward-scattering amplitude proportional to $\vec{s}_{n} \cdot\left(\vec{k}_{n} \times \vec{I}\right)$, where $\vec{s}_{n}$ is the neutron spin, $\vec{k}_{n}$ is the neutron momentum, and $\vec{I}$ is the spin of the nucleus. The highly excited states formed in these heavy compound nuclei provide a distinct landscape for study compared to the ground states of nucleons and nuclei that are probed by electric dipole moment experiments. At a particular $p$-wave resonance, the ratio of the P-odd and T-odd amplitude to the P-odd amplitude is notably insensitive to complicated unknown properties of the involved compound resonant states. In the case of elastic neutron-scattering in the forward direction, the differences in cross-section for the normal and time-reversed processes are proportional to the differences in scattering amplitude, which does not change since the state of the polarized target is the same, and through the optical theorem the imaginary part of the forward-scattering amplitude is directly related to the cross-section. This implies that in such a system, one can realize a sensitive null test for time-reversal invariance that is free from the effects of final-state interactions in principle [114-116].

Typical weak NN interaction amplitudes are on the order of $10^{-7}$ relative to the strong interaction; however, amplifications of $10^{6}$ giving a relative strength of $10^{-1}$ have already been observed [75] in measurements of $\Delta \sigma_{P}$ several heavy nuclei, including some at $p$-wave resonances in the few $\mathrm{eV}$ energy range, such as ${ }^{139} \mathrm{La}[117],{ }^{131} \mathrm{Xe}[118,119]$, ${ }^{81} \mathrm{Br}$ [120-122], and ${ }^{117} \mathrm{Sn}$. This amplification resulting from the mixing of nearby $\mathrm{s}$ and p-wave resonances was predicted theoretically before it was measured, and the same amplification factor of resonances applies to an amplitude that is both P-odd and T-odd up to factors of order unity. Although the nuclear states involved are extremely complicated at the level of the many-body nuclear wave functions, one can form a dimensionless ratio $\lambda_{P T}=\frac{\Delta \sigma_{T P}}{\Delta \sigma_{P}}=\kappa(J) \frac{\left\langle\phi_{p}\left|V_{P T}\right| \phi_{s}\right\rangle}{\left\langle\phi_{p}\left|V_{P}\right| \phi_{s}\right\rangle}$ of the T-odd, P-odd asymmetry $\Delta \sigma_{T P}$ of interest to the measured P-odd asymmetry $\Delta \sigma_{P}$ at the position of the enhanced p-wave resonance energy. In this expression, the ratio $\frac{\left\langle\phi_{p}\left|V_{P T}\right| \phi_{s}\right\rangle}{\left\langle\phi_{p}\left|V_{P}\right| \phi_{s}\right\rangle}$ of the matrix elements of the P-odd and T-odd interaction to the P-odd interaction is between the same pair of $\mathrm{s}$ and $\mathrm{p}$ wave resonance states $\mid \phi_{s}>$ and $\mid \phi_{p}>$, and a spin-weighted sum of resonance partial widths $\kappa(J)$ which can be determined experimentally using $(n, \gamma)$ spectroscopy. As this ratio involves expectation values using identical compound nuclear wave functions, a clean theoretical interpretation can be provided.

Using a ${ }^{139}$ La target on a beamline at a MW-class short pulse neutron spallation source, a statistical uncertainty could be achieved after $10^{7} \mathrm{~s}$ of data acquisition yielding a ratio $\lambda_{P T}$ with $1 \times 10^{-4}$ to $1 \times 10^{-5}$ sensitivity, translating into an improved sensitivity of P-odd and T-odd neutron-nucleus interactions of at least an order of magnitude [103,115,116,123,124]. 
The $0.7 \mathrm{eV}$ resonance in ${ }^{139} \mathrm{La}$ has a relatively large P-odd longitudinal asymmetry of 9.5\% [117] and is therefore a good candidate for this search. $\kappa$ has been constrained recently in ${ }^{139} \mathrm{La} \mathrm{[125]} \mathrm{to} \mathrm{be} \mathrm{at} \mathrm{least} \mathrm{of} \mathrm{order} \mathrm{1,} \mathrm{and} \mathrm{ongoing} \mathrm{experiments} \mathrm{at} \mathrm{JPARC} \mathrm{will} \mathrm{soon}$ measure $\kappa$ in other Neutron OPtics time-reversal EXperiment (NOPTREX) candidate nuclei. Groups at KEK [126], Kyoto University [127], and PSI [128] have achieved a substantial 50\% polarization of ${ }^{139}$ La nuclei in lanthanum aluminate crystals in volumes as large as $10 \mathrm{~cm}^{3}$, enough for a time-reversal violation experiment using neutron optics, and R\&D to polarize ${ }^{81} \mathrm{Br}[129],{ }^{131} \mathrm{Xe}$ [130] and ${ }^{117} \mathrm{Sn}$ [131] is in progress. Ongoing R\&D on high phase-space acceptance supermirror neutron optics has the potential to improve the statistical sensitivity in the future by another order of magnitude.

This estimated sensitivity accessible today is comparable to that being proposed for the next-stage neutron EDM searches. However, as the neutron-nucleus system possesses interactions not present in the single neutron system involved in nEDM searches, it is quite possible that $\mathrm{P}$ and $\mathrm{T}$ violation might be seen in one of these observables, but not the other [132-134]. In particular, the NOPTREX observable is sensitive to axion-like particles $[135,136]$. It is therefore very important to pursue such a search if one can suppress the potential sources of systematic error. As no such polarized neutron optics search for P-odd and T-odd interactions has ever been conducted, the first real experiment will represent a pioneering effort.

The bright pulsed sources of epithermal neutrons at MW-class spallation neutron facilities like SNS and JSNS have enough intensity at $\mathrm{eV}$ energies to reach the statistical accuracy required for a sensitive search. The separation of neutron energies using timeof-flight measurements at the pulsed sources also enables a careful study of systematic errors by looking above and below the resonance energy for both transmitted and scattered neutrons. Existing technology at $\mathrm{eV}$ neutron polarization using polarized ${ }^{3} \mathrm{He}$ neutron spin filters suffice for the measurement.

Birefringent neutron optical devices recently developed for neutron spectroscopy can convert the NOPTREX experimental apparatus into a spin-path interferometer, similar to the Ramsey separated oscillatory field configuration used in electric dipole moment searches but operating with paths separated in space rather than in time. These devices were recently used to entangle the neutron spin and position or the neutron spin, position, and energy variables into Bell and Greenberger-Horne-Zeilinger (GHZ) states, whose degree of quantum entanglement was quantified by measuring the appropriate Bell and GHZ entanglement witnesses $[84,85,137]$. The correlation observables in this experiment took the largest possible value allowed by quantum mechanics despite the passage of the polarized neutrons through macroscopic amounts of matter. The small decoherence of the transmitted neutron state confirmed by this work implies that neutron interferometric methods based on this technology can be applied to NOPTREX to help isolate the P-odd/Todd signal of interest from many possible sources of systematic error and help ensure that the neutron optical T-odd null test condition is satisfied.

Searches for new sources of $\mathrm{T}$ violation using nucleons and nuclei are, therefore, not only important because of their high sensitivity to specific types of BSM physics, but are also timely because they can address open questions in cosmology. Continued studies can lead to relatively near-term progress both in our understanding of the baryon asymmetry by probing many possibilities at and near the electroweak scale and also possibly in the dark matter problem through axions.

Axion-like particles (ALPs) generate a P-odd and T-odd spin-dependent Yukawa potential proportional to $g_{s} g_{p} \vec{\sigma} \cdot \vec{r}$. Different experiments are sensitive to different ALP masses. Neutron EDM searches using ultra-cold neutrons are typically sensitive to ALP masses in the $\mu \mathrm{eV}$ range corresponding to the distance scale that ultra-cold neutrons penetrate into the material walls of the storage container. Neutron dynamical diffraction can be sensitive to ALPs with meV to $\mathrm{eV}$ masses. Parity-odd and time-reversal-odd forward-scattering is sensitive through the optical theorem to all ALP masses above that set by the length scale of the polarized nuclear target. Very stringent upper bounds on light 
mass ALPs from astrophysics already exist: $g_{s}$ limits from tests of gravity are combined with $g_{p}$ limits from analysis of stellar cooling dynamics. However, these constraints are typically applicable for very light-mass ALPs, and are more indirect compared to laboratory experiments.

Recent theoretical work has explored the relationship between ALP constraints from upper bounds on electric dipole moments and those from laboratory experiments looking for a $\vec{\sigma} \cdot \vec{r}$ interaction [135]. This work showed that EDM constraints dominate over fifthforce limits by several orders of magnitude for axion-mediated interactions, but that for generic ALPs bounds from laboratory experiments are more stringent than EDM limits over a wide parameter range. The sensitivity of polarized neutron optical transmission experiments through polarized nuclei to generic ALPs has been evaluated recently [136]. Electric dipole moment limits combined with atomic theory calculations give a relatively weak, indirect limit on $\lambda_{P T}<10^{-3}$. We conclude that windows of opportunity exist for polarized neutron optical transmission experiments through polarized nuclei to search for and possibly discover generic ALPs.

\section{Time Variation of QCD Parameters}

Ever since Dirac speculated that one or more of the fundamental constants of nature might somehow be coupled to the expansion of the universe and therefore possess some small but nonzero time variation, physicists have taken advantage of all possible opportunities to test the idea. It is a useful exercise quite apart from the desire to test any specific theory or mechanism. The analyses we conduct to try to test cosmological dynamics such as Big Bang nucleosynthesis naturally assume that no such time-dependent evolution of properties has taken place, and one can interpret its success as placing severe limits on any such variation. In the case of the electromagnetic coupling constant $\alpha$, one can of course perform "ancient" spectroscopy by looking at atomic transitions from highly-redshifted astrophysical systems. The precision achievable now by atomic spectroscopy is so sensitive that one can now foresee the ability to make this test now, over timescales, accessible in the laboratory. Recent activity on this front has occasionally veered close to claiming evidence for a time variation of $\alpha$, but to the knowledge of these authors, there is no such evidence at the moment.

One can of course ask the same question about other fundamental constants. Another obvious coupling to look at is the QCD gauge coupling constant $\alpha_{S}$. Certainly the direct measurement of $\alpha_{S}$ from laboratory experiments is far too crude for one to entertain any hope to address such a cosmological issue. Furthermore, the more appropriate quantity to constrain is a dimensionless ratio like $X=m / \Lambda_{Q C D}$, where $m$ is a light quark mass and $\Lambda_{Q C D}$ is the non-perturbative strong interaction scale. To our knowledge, the most stringent bound on a possible time variation of $X$ comes from an ex-post-facto analysis of the observed isotopic distributions of the ores at the famous Oklo uranium mining complex in Gabon [138]. Plausible arguments and analyses indicate that a natural nuclear reactor operated in this area about two billion years ago. If one accepts this hypothesis, then one can use the proximity of the resonance energy and width of the isotope ${ }^{149} \mathrm{Sm}$ in these ores to the thermal moderated neutron energy spectrum in the reactor to place an interesting upper bound on the time variation of $X$. The point is that this isotope has a sharp near-threshold neutron-nucleus resonance with a large neutron absorption. If this resonance was in the wrong place two billion years earlier, which can happen as $X$ changes with time, it could have rapidly absorbed the thermalized neutrons and either stopped the ancient reactor or greatly modified the resulting distributions of the ${ }^{149} \mathrm{Sm} /{ }^{150} \mathrm{Sm}$ isotope ratio found in the ores. This argument places an upper bound on the time variation of $\frac{d X}{d t}<2 \times 10^{-18} /$ year [139]. Analysis of the ${ }^{176} \mathrm{Lu} /{ }^{175} \mathrm{Lu}$ isotopic ratio in the cores could also be used to check the internal consistency of the Oklo model [140].

It is very difficult to imagine how a laboratory experiment might compete with this bound. However, sensitive laboratory measurements are still very interesting for the reasons discussed above. One physical system discussed for this purpose is the transition 
between the ground and the first excited states in the ${ }^{229} \mathrm{Th}$ nucleus $[139,141,142]$ in view of its potential use as a nuclear clock due to its extremely low-energy of a few $\mathrm{eV}$ for its first nuclear excited state. The transition energy in this system is very sensitive to both time variations in the strong and electromagnetic interactions.

We would like to call attention to another system which might be a good choice for a laboratory search and which would be dominantly sensitive to strong interaction variations, thereby complementing the ${ }^{229} \mathrm{Th}$ nuclear clock approach. Let a polarized neutron beam be transmitted through a medium of heavy nuclei with neutron-nucleus resonances. Following the treatments performed for the Oklo bounds based on a nuclear theory analyses of the relationship of the fractional change in the strong couplings to the shift in the energy of a near-threshold neutron-nucleus resonance of $\frac{1}{\alpha_{S}} \frac{d \alpha_{S}}{d t}=\Delta_{r} / M$, where $\Delta_{r}$ is the shift in resonance energy and $M=1 \mathrm{MeV}$ is the relevant nuclear energy scale determined from theory calculations, we expect that a neutron transmission spectroscopy experiment sensitive to a $1 \mathrm{neV}$ shift of the resonance width repeated over the course of one year could sense a fractional variation in $\frac{1}{\alpha_{S}} \frac{d \alpha_{S}}{d t}$ of $10^{-15} / \mathrm{yr}$.

Neutron spin echo spectrometers for slow neutrons routinely place the neutron beam in a mode-entangled coherent superposition state of the form $|\uparrow\rangle|E\rangle+|\downarrow\rangle|E+\Delta\rangle$ with $\Delta<1 \mu \mathrm{eV}$, where $|\uparrow\rangle$ and $|\downarrow\rangle$ indicate neutron spin states. The same type of beam can also be made for $\mathrm{eV}$ energy neutron beams (although it seems it has not been done before). If the nucleus is unpolarized so that the spin-dependent neutron-nucleus strong interaction effects are not manifest, and if the resonance does not possess any amplification of parity violation which could give a spin-dependent phase shift, then one can imagine doing a sensitive transmission experiment through such a medium with this mode-entangled beam. Before proceeding further one should conduct a serious systematic error analysis and also perform a theoretical analysis of this system in terms of $X$.

One would also need to be sure that a variation was not due to the variation of some other fundamental coupling of Nature. To our knowledge the best present upper bounds on a possible time variation of the Fermi weak interaction constant $G_{F}$ come from an analysis [143] of the decay curves from Type $1 \mathrm{~A}$ supernovas, of order $10^{-9} / \mathrm{yr}$, and the Oklo analysis of the isotopic abundance of ${ }^{149} \mathrm{Sm}$, one of the heavy nuclei which possesses a large neutron resonance near threshold, which gives about a $10^{-11} / \mathrm{yr}$ limit. Neither of these are laboratory-based bounds.

\section{Conclusions}

In this work, we have tried to give an overview of recent neutron-based experimental searches for new weakly-coupled interactions, some of which are also of interest for various types of particle dark-matter candidates, with special attention to searches for possible exotic gravity Yukawa interactions with ranges in the sub-micron regime. Many of these initial searches were conducted through small modifications of existing apparatus or reanalysis of existing experimental data. Although there is still room for such contributions, experimental work in this area is now increasingly transitioning to optimized designs which attempt to maximize the sensitivity to specific types of interactions, while making the best possible use of intense neutron beams and taking full advantage of new systems which can amplify the effects of such interactions in accessible neutron observables. We showed that there is great promise for further progress in this class of searches by using polarized epithermal neutrons and the properties of neutron-nucleus resonances to amplify select variables. We also gave an example of a possible laboratory-based neutron experiment to search for a possible time-variation of the strong interaction ratio $X=m / \Lambda_{Q C D}$, where $m$ is a light quark mass and $\Lambda_{Q C D}$ is the non-perturbative strong interaction scale.

Author Contributions: Investigation, W.M.S., B.H. and C.H.; Methodology, W.M.S., B.H. and C.H.; Project Administration, W.M.S., B.H. and C.H.; Supervision, W.M.S., B.H. and C.H.; Visulaization: B.H. and C.H.; Writing-original draft, W.M.S., B.H. and C.H.; Writing-review and editing, W.M.S., B.H. and C.H. All authors have read and agreed to the published version of the manuscript. 
Funding: NSF PHY-1913789, National Institute of Standards and Technology.

Institutional Review Board Statement: Not applicable.

Informed Consent Statement: Not applicable.

Data Availability Statement: The data used to make Figure 1 is available at https: / zenodo.org/ record/5080965\#.YUOf3rhKi70 (accessed on 13 December 2021) in the file Fig3.xls.

Acknowledgments: W. M. Snow acknowledges support from US National Science Foundation grant PHY-1913789 and the Indiana University Center for Spacetime Symmetries. C. Haddock and B. Heacock acknowledge support from the National Institute of Standards and Technology.

Conflicts of Interest: The authors declare no conflict of interest.

\section{References}

1. Nico, J.S.; Snow, W.M. Fundamental Neutron Physics. Ann. Rev. Nucl. Part. Sci. 2005, 55, 27. [CrossRef]

2. Abele, H. The neutron. Its properties and basic interactions. Prog. Part. Nucl. Phys. 2008, 60, 1. [CrossRef]

3. Dubbers, D.; Schmidt, M.G. The Neutron and Its Role in Cosmology and Particle Physics. Rev. Mod. Phys. $2011,83,1111$. [CrossRef]

4. Pignol, G. Probing Dark Energy Models with Neutrons. Int. J. Mod. Phys. A 2015, 30, 1530048. [CrossRef]

5. Leeb, H.; Schmiedmayer, J. Constraint on hypothetical light interacting bosons from low-energy neutron experiments. Phys. Rev. Lett. 1992. 68, 1472. [CrossRef]

6. Baessler, S.; Nesvizhevsky, V.V.; Protasov, K.V.; Voronin, A.Y. Constraint on the coupling of axionlike particles to matter via an ultracold neutron gravitational experiment. Phys. Rev. D 2007, 75, 075006. [CrossRef]

7. Serebrov, A. New constraints for CP-violating forces between nucleons in the range 1 micrometer to 1 centimeter. Phys. Lett. $B$ 2009, 680, 423-427. [CrossRef]

8. Ignatovich, V.K.; Pokotilovski, Y.N. Limits on a nucleon-nucleon monopole-dipole coupling from spin relaxation of polarized ultra-cold neutrons in traps. Eur. Phys. J. C 2009, 64, 19. [CrossRef]

9. Piegsa, F.M.; Pignol, G. Limits on the Axial Coupling Constant of New Light Bosons. Phys. Rev. Lett. 2012, 108, 181801. [CrossRef]

10. Yan, H.; Snow, W.M. New Limit on Possible Long-Range Parity-Odd Interactions of the Neutron from Neutron-Spin Rotation in Liquid ${ }^{4}$ He. Phys. Rev. Lett. 2013, 110, 082003. [CrossRef]

11. Lehnert, R.; Snow, W.M.; Yan, H. A first experimental limit on in-matter torsion from neutron spin rotation in liquid ${ }^{4}$ He. Phys. Lett. B 2014, 730, 353; Erratum in Phys. Lett. B 2015, 744, 415. [CrossRef]

12. Jenke, T.; Cronenberg, G.; Burgdorfer, J.; Chizhova, L.A.; Geltenbort, P.; Ivanov, A.N.; Lauer, T.; Lins, T.; Rotter, S.; Saul, H.; et al. Gravity Resonance Spectroscopy Constrains Dark Energy and Dark Matter Scenarios. Phys. Rev. Lett. 2014, 112, 151105. [CrossRef] [PubMed]

13. Lemmel, H.; Brax, P.; Ivanov, A.N.; Jenke, T.; Pignol, G.; Pitschmann, M.; Potocar, T.; Wellenzohn, M.; Zawisky, M.; Abele, H. Neutron interferometry constrains dark energy chameleon fields. Phys. Lett. B 2015, 743, 310. [CrossRef]

14. Li, K.; Arif, M.; Cory, D.G.; Haun, R.; Heacock, B.; Huber, M.G.; Nsofini, J.; Pushin, D.A.; Saggu, P.; Sarenac, D.; et al. Neutron limit on the strongly-coupled chameleon field. Phys. Rev. D 2016, 93, 062001. [CrossRef]

15. Lehnert, R.; Snow, W.M.; Xiao, Z.; Xu, R. Constraining spacetime nonmetricity with neutron spin rotation in liquid ${ }^{4}$ He. Phys. Lett. B 2017, 772, 865. [CrossRef]

16. Haddock, C.; Amadio, J.; Anderson, E.; Barron-Palos, L.; Crawford, B.; Crawford, C.; Esposito, D.; Fox, W.; Francis, I.; Fry, J.; et al. A search for possible long range spin dependent interactions of the neutron from exotic vector boson exchange. Phys. Lett. B 2018, 783, 227. [CrossRef]

17. Cronenberg, G.; Brax, P.; Filter, H.; Geltenbort, P.; Jenke, T.; Pignol, G.; Pitschmann, P.; Thalhammer, M.; Abele, H. Acoustic Rabi oscillations between gravitational quantum states and impact on symmetron dark energy. Nat. Phys. 2018, 14, 1022. [CrossRef]

18. Sponar, S.; Sedmik, R.I.P.; Pitschmann, M.; Abele, H.; Hasegawa, Y. Tests of fundamental quantum mechanics and dark interactions with low-energy neutrons. Nat. Rev. Phys. 2021, 3, 309. [CrossRef]

19. Safronova, M.S.; Budker, D.; DeMille, D.; Kimball, D.F.J.; Derevianko, A.; Clark, C.W. Search for new physics with atoms and molecules. Rev. Mod. Phys. 2018, 90, 025008. [CrossRef]

20. Leitner, J.; Okubo, S. Parity, Charge Conjugation, and Time Reversal in the Gravitational Interaction. Phys. Rev. 1964, 136, B1542. [CrossRef]

21. Hill, C.T.; Ross, G.G. Models and new phenomenological implications of a class of pseudo-Goldstone bosons. Nucl. Phys. B 1988, 311, 253. [CrossRef]

22. Jaeckel, J.; Ringwald, A. The Low-Energy Frontier of Particle Physics. Annu. Rev. Nucl. Part Sci. 2010, 60, 405. [CrossRef]

23. Arvanitaki, A.; Dimopoulos, S.; Dubovsky, S.; Kapoler, N.; March-Russell, J. String Axiverse. Phys. Rev. D 2010, 81, 123530. [CrossRef]

24. Olive, K.A.; Particle Data Group. Review of Particle Physics. Chin. Phys. C 2014, 38, 090001. [CrossRef]

25. Weinberg, S. Approximate Symmetries and Pseudo-Goldstone Bosons. Phys. Rev. Lett. 1972, 29, 1698. [CrossRef] 
26. Dobrescu, B.A. Massless gauge bosons other than the photon. Phys. Rev. Lett. 2005, 94, 151802. [CrossRef] [PubMed]

27. Appelquist, T.; Dobrescu, B.A.; Hopper, A.R. Nonexotic neutral gauge bosons. Phys. Rev. D 2003, 68, 035012. [CrossRef]

28. Dobrescu, B.; Mocioiu, I. Spin-dependent macroscopic forces from new particle exchange. J. High Energy Phys. 2006, 11, 005. [CrossRef]

29. Fadeev, P.; Stadnik, Y.V.; Ficek, F.; Kozlov, M.G.; Flambaum, V.V.; Budker, D. Revisiting spin-dependent forces mediated by new bosons. Phys. Rev. A 2019, 99, 022113. [CrossRef]

30. Raffelt, G.G.; Weiss, A. Red giant bound on the axion-electron coupling reexamined. Phys. Rev. D 1995, 51, 1495. [CrossRef]

31. Raffelt, G.G. Stars as Laboratories for Fundamental Physics; University of Chicago Press: Chicago, IL, USA, 1995.

32. Raffelt, G.G. Limits on a CP-violating scalar axion-nucleon interaction. Phys. Rev. D 2012, 86, 015001. [CrossRef]

33. Adelberger, E.G.; Wagner, T.A. Improved limits on long-range parity-odd interactions of the neutron. Phys. Rev. D 2014, 88, 031101(R). [CrossRef]

34. Jain, P.; Mandal, S. Evading the astrophysical limits on light pseudoscalars. Int. J. Mod. Phys. D 2006, 15, 2095. [CrossRef]

35. Costantino, A.; Fichet, S.; Tanedo, P. Exotic Spin-Dependent Forces from a Hidden Sector. J. High Energy Phys. $2020,148$. [CrossRef]

36. Fichet, S. Quantum Forces from Dark Matter and Where to Find Them. Phys. Rev. Lett. 2018, 120, 131801. [CrossRef]

37. Brax, P.; Fichet, S.; Pignol, G. Bounding quantum dark forces. Phys. Rev. D 2018, 97, 115034. [CrossRef]

38. Arkani-Hamed, N.; Dimopoulos, S.; Dvali, G. The Hierarchy Problem and New Dimensions at a Millimeter. Phys. Lett. B 1998, 429, 263. [CrossRef]

39. Adelberger, E.G.; Heckel, B.R.; Nelson, A.E. Tests of the Gravitational Inverse-Square Law. Annu. Rev. Nucl. Part. Sci. 2003, 53, 77. [CrossRef]

40. Frank, A.; Isacker, P.V.; Gomez-Camacho, J. Probing additional dimensions in the universe with neutron experiments. Phys. Lett. B 2004, 582, 15. [CrossRef]

41. Adelberger, E.G.; Gundlach, J. H.; Heckel, B. R.; Hield, S.; Schlamminger, S. Torsion balance experiments: A low-energy frontier of particle physics. Prog. Part. Nucl. Phys. 2009, 62, 102. [CrossRef]

42. Murata, J.; Tanaka, S. A review of short-range gravity experiments in the LHC era. Class. Quantum Grav. 2015, $32,33001$. [CrossRef]

43. Nesvizhevsky, V.V.; Pignol, G.; Protasov, K.V. Neutron scattering and extra-short-range interactions. Phys. Rev. D 2008, 77, 034020. [CrossRef]

44. Kamiya, Y.; Itagaki, K.; Tani, M.; Kim, G. N.; Komamiya, S. Constraints on New Gravitylike Forces in the Nanometer Range. Phys. Rev. Lett. 2015, 114, 161101. [CrossRef]

45. Haddock, C.; Oi, N.; Hirota, K.; Ino, T.; Kitaguchi, M.; Matsumoto, S.; Mishima, K.; Shima, T.; Shimizu, H.M.; Snow, W.M.; et al. Search for deviations from the inverse square law of gravity at nm range using a pulsed neutron beam. Phys. Rev. D 2018, 97, 062002. [CrossRef]

46. Pokotilovski, Y.N. Constraints on New Interactions from Neutron Scattering Experiments. Phys. At. Nucl. 2006, 69, 924. [CrossRef]

47. Kamyshkov, Y.; Tithof, J.; Vysotsky, M. Bounds on new light particles from high-energy and very small momentum transfer np elastic scattering data. Phys. Rev. D 2008, 78, 114029. [CrossRef]

48. Salumbides, E.J.; Schellekens, A.N.; Gato-Rivera, B.; Ubachs, W. Constraints on extra dimensions from precision molecular spectroscopy. New J. Phys.2015, 17, 033015. [CrossRef]

49. Heacock, B.; Fujiie, T.; Haun, R.W.; Henins, A.; Hosobata, T.; Huber, M.G.; Kitaguchi, M.; Pushin, D.A.; Shimizu, H.; Takeda, M.; et al. Pendellosung interferometry probes the neutron charge radius, lattice dynamics, and fifth forces. Science 2021, $373,1239$. [CrossRef] [PubMed]

50. Klimchitskaya, G.L.; Kuusk, P.; Mostepanenko, V.M. Constraints on non-Newtonian gravity and axionlike particles from measuring the Casimir force in nanometer separation range. Phys. Rev. D 2020, 101, 056013. [CrossRef]

51. Voronin, V.V.; Fedorov, V.V.; Kuznetsov, I.A. Neutron diffraction test on spin-dependent short range interaction. JETP Lett. 2009, 90, 5. [CrossRef]

52. Kopecky, S.; Harvey, J.A.; Hill, N.W.; Krenn, M.; Pernicka, M.; Riehs, P.; Steiner, S. Neutron charge radius determined from the energy dependence of the neutron transmission of liquid ${ }^{208} \mathrm{~Pb}$ and ${ }^{209} \mathrm{Bi}$. Phys. Rev. C 1997, 56, 2229. [CrossRef]

53. Itoh, S.; Nakaji, M.; Uchida, Y.; Kitaguchi, M.; Shimizu, M. Pendellosung interferometry by using pulsed neutrons. Nucl. Instrum. Methods Phys. Res. Sect. 2018, 908, 78. [CrossRef]

54. Horne, M.A.; Finkelstein, K.D.; Shull, C.G.; Zeilinger, A.; Bernstein, H.J. Neutron spin-Pendellosung resonance. Phys. B 1988, 151, 189. [CrossRef]

55. Gentile, T.R.; Huber, M.G.; Koetke, D.D.; Peshkin, M.; Arif, M.; Dombeck, T.; Hussey, D.S.; Jacobson, D.L.; Nord, P.; Pushin, D.A.; et al. Direct observation of neutron spin rotation in Bragg scattering due to the spin-orbit interaction in silicon. Phys. Rev. C 2019, 100, 034005. [CrossRef]

56. Jenke, T. Geltenbort, P.; Lemmel, H.; Abele, H. Realization of a gravity-resonance-spectroscopy technique. Nat. Phys. 2011, 7, 468. [CrossRef]

57. Abele, H.; Jenke, T.; Leeb, H.; Schmiedmayer, J. Ramsey's method of separated oscillating fields and its application to gravitationally induced quantum phase shifts. Phys. Rev. D 2010, 81, 065019. [CrossRef] 
58. Sedmik, R.I.P.; Bosina, J.; Achatz, L.; Geltenbort, P.; Heis, M.; Ivanov, A.N.; Jenke, T.; Micko, J.; Pitschmann, M.; Rechberger, T.; et al. Proof of principle for Ramsey-type gravity resonance spectroscopy with qBounce. EPJ Web Conf. 2019, 219, 05004. [CrossRef]

59. Ivanov, A.N.; Hollwieser, R.; Jenke, T.; Wellenzohn, M.; Abele, H. Influence of the chameleon field potential on transition frequencies of gravitationally bound quantum states of ultracold neutrons. Phys. Rev. D 2013, 87, 105013. [CrossRef]

60. Ivanov, A.N.; Cronenberg, G.; Hollwieser, R.; Jenke, T.; Pitschmann, M.; Wellenzohn, M.; Abele, H. Exact solution for chameleon field, self-coupled through the Ratra-Peebles potential with $n=1$ and confined between two parallel plates. Phys. Rev. D 2016, 94, 085005. [CrossRef]

61. Klimchitskaya, G.L.; Mostepanenko, V. M.; Sedmik, R. I. P.; Abele, H. Prospects for Searching Thermal Effects, Non-Newtonian Gravity and Axion-Like Particles: Cannex Test of the Quantum Vacuum. Symmetry 2019, 11, 407. [CrossRef]

62. Abele, H.; Baeßler, S.; Westphal, A. Quantum states of neutrons in the gravitational field and limits for non-Newtonian interaction in the range between 1 micron and 10 microns. Lect. Notes Phys. 2003, 631, 355.

63. Ivanov, A.N.; Wellenzohn, M.; Abele, H. Quantum gravitational states of ultracold neutrons as a tool for probing of beyondRiemann gravity. Phys. Lett. B 2021, 822, 136640. [CrossRef]

64. Schmidt-Wellenburg, P.; Andersen, K.H.; Courtois, P.; Kreuz, M.; Mironov, S.; Nesvizhevsky, V.V.; Pignol, G.; Protasov, K.V.; Soldner, T.; Vezzu, F.; et al. Ultracold-neutron infrastructure for the gravitational spectrometer GRANIT. Nucl. Instr. Meth. A 2009, 611, 267. [CrossRef]

65. Nesvizhevsky, V.V.; Borner, H.G.; Petukhov, A.K.; Abele, H.; Baessler, S.; Rue, F.J.; Sto ferle, T.; Westphal, A.; Gagarski, A.M.; Petrov, G.A.; et al. Quantum states of neutrons in the Earth's gravitational field. Nature 2002, 415, 297. [CrossRef] [PubMed]

66. Kreuz, M.; Nesvizhevsky, V.V.; Schmidt-Wellenburg, P.; Soldner, T.; Thomas, M.; Börner, H.G.; Naraghi, F.; Pignol, G.; Protasov, K.V.; Rebreyend, D.; et al. A method to measure the resonance transitions between the gravitationally bound quantum states of neutrons in the GRANIT spectrometer. Nucl. Instrum. Methods Phys. Res. A 2009, 611, 326. [CrossRef]

67. Piegsa, F.M.; Fertl, M.; Ivanov, S.N.; Kreuz, M.; Leung, K.K.H.; Schmidt-Wellenburg, P.; Soldner, T.; Zimmer, O. New source for ultracold neutrons at the Institut Laue-Langevin. Phys. Rev. C2014, 90, 015501. [CrossRef]

68. Gudkov, V.P.; Opat, G.I.; Klein, A.G. Neutron reflection interferometry: Physical principles of surface analysis with phase information. J. Phys. Condens. Matter 1993, 5, 9013. [CrossRef]

69. Pokotolovskii, Y.N. Strongly coupled chameleon fields: Possible test with a neutron Lloyd's mirror interferometer. Phys. Lett. B 2013, 719, 341. [CrossRef]

70. Nakajima, K.; Kawakita, Y.; Itoh, S.; Abe, J.; Aizawa, K.; Aoki, H.; Endo, H.; Fujita, M.; Funakoshi, K.; Gong, W.; et al. Materials and Life Science Experimental Facility (MLF) at the Japan Proton Accelerator Research Complex II: Neutron Scattering Instruments. Quantum Beam Sci. 2017, 1, 9. [CrossRef]

71. Coulter, K.P.; Chupp, T.E.; McDonald, A.B.; Bowman, C.D.; Bowman, J.D.; Szymanski, J.J.; Yuan, V.; Catee, G.D.; Benton, D.R.; Earle, E.D. Neutron polarization with a polarized ${ }^{3}$ He spin filter. Nucl. Inst. Meth. A 1990, 288, 463. [CrossRef]

72. Taran, Y.V.; Shaporo, F.L. Some methods for polarizing the intermediate-energy neutrons and analyzing their polarization. Zh. Eksp. Theo. Fiz. 1963, 44, 2185.

73. Haag, M.; Brandt, B.; Eichhirn, T.R.; Hautle, P.; Wenckenbach, T. Spin filtering neutrons with a proton target dynamically polarized using photo-excited triplet states. Nucl. Inst. Meth. 2012, 678, 91. [CrossRef]

74. Takada, S.; Tateishi, K.; Wakabayashi, Y.; Ikeda, Y.; Yoshioka, T.; Otake, Y.; Uesaka, T. Polarized proton spin filter for epithermal neutrons based on dynamic nuclear polarization using photo-excited triplet electron spins. Prog. Theo. Exp. Phys. 2020, $123 \mathrm{G} 01$. [CrossRef]

75. Mitchell, G.E.; Bowman, J.D.; Weidenmuller, H.A. Parity violation in the compound nucleus. Rev. Mod. Phys. 1999, 71, 445. [CrossRef]

76. Mitchell, G.E.; Bowman, J.D.; Penttila, S.I.; Sharapov, E.I.; Parity violation in compound nuclei: Experimental methods and recent results. Phys. Rep. 2001, 354, 157. [CrossRef]

77. Blyth, D.; Fry, J.; Fomin, N.; Alarcon, R.; Alonzi, L.; Askanazi, E.; Baessler, S.; Balascuta, S.; Barron-Palos, L.; Barzilov, A.; et al. (NPDGamma Collaboration), First Observation of P-odd $\gamma$ Asymmetry in Polarized Neutron Capture on Hydrogen. Phys. Rev. Lett. 2018, 121, 242002. [CrossRef] [PubMed]

78. Gericke, M.T.; Baessler, S.; ; Barron-Palos, L.; Birge, N.; Bowman, J.D.; Calarco, J.; Cianciolo, V.; Coppola, C.E.; Crawford, C.B.; Fomin, N.; et al. (nHe3 Collaboration), First Precision Measurement of the Parity Violating Asymmetry in Cold Neutron Capture on ${ }^{3}$ He. Phys. Rev. Lett. 2020, 125, 131803. [CrossRef] [PubMed]

79. Altuntas, E.; Ammon, J.; Cahn, S.B.; DeMille, D. Demonstration of a Sensitive Method to Measure Nuclear-Spin-Dependent Parity Violation. Phys. Rev. Lett. 2018, 120, 142501. [CrossRef] [PubMed]

80. Flambaum, V.V.; Vorov, O.K. Matrix elements between nuclear compound states and dynamical enhancement of the weak interaction. Phys. Rev. Lett. 1993, 70, 4051. [CrossRef] [PubMed]

81. Tomsovic, S.; Johnson, M.B.; Hayes, A.C.; Bowman, J.D. Statistical theory of parity nonconservation in compound nuclei. Phys. Rev. C 2000, 62, 054607. [CrossRef]

82. Flambaum, V.V.; Mansour, A.J. Parity and Time Reversal Invariance Violation in Neutron-Nucleus Scattering. arXiv 2021, arXiv:2111.02037.

83. Hayes, A.C.; Zanini, L. Parity violation in partial neutron capture reactions. Phys. Rev. C 2002, 65, 058501. [CrossRef] 
84. Shen, J.; Kuhn, S.; Baxter, D.; Dalgleish, R.; Li, F.; Lu, S.; Ortiz, G.; Plomp, J.; Parnell, S.; Pynn, R.; et al. Unveiling contextual realities by microscopically entangling a neutron. Nat. Comm. 2020, 11, 930. [CrossRef] [PubMed]

85. Kuhn, S.J.; McKay, S.; Shen, J.; Geerits, N.; Dalgliesh, R.; Dees, E.; Irfan, A.A.M.; Li, F.; Lu, S.; Vangelista, V.; et al. Neutron State Entanglement with Overlapping Paths. Phys. Rev. Res. 2021, 3, 023227. [CrossRef]

86. Li, F.; Parnell, S.R.; Bai, H.; Yang, W.; Hamilton, W.A.; Maranville, B.B.; Ashkar, R.; Baxter, D.V.; Cremer, J.T.; Pynn, R. Spin echo modulated small-angle neutron scattering using superconducting magnetic Wollaston prisms. J. Appl. Cryst. 2016, 49, 55. [CrossRef]

87. Clark, C.W.; Barankov, R.; Huber, M.G.; Arif, M.; Cory, D.G.; Pushin, D.A. Controlling neutron orbital angular momentum. Nature 2015, 525, 504. [CrossRef] [PubMed]

88. Nsofini, J.; Sarenac, D.; Wood, C.J.; Cory, D.G.; Arif, M.; Clark, C.W.; Huber, M.G.; Pushin, D.A. Spin-orbit states of neutron wave packets. Phys. Rev. A 2016, 94, 013605. [CrossRef]

89. Sarenac, D.; Nsofini, J.; Hincks, I.; Arif, M.; Clark, C.W.; Cory, D.G.; Huber, M.G.; Pushin, D.A. Methods for preparation and detection of neutron spin-orbit states. New J. Phys. 2018, 20, 103012. [CrossRef]

90. Cappelletti, R.; Jach, T.; Vinson, J. Intrinsic Orbital Angular Momentum States of Neutrons. Phys. Rev. Lett. 2018, 120, 090402. [CrossRef] [PubMed]

91. Sarenac, D.; Kapahi, C.; Chen, W.; Clark, C.W.; Cory, D.G.; Huber, M.G.; Taminiau, I.; Zhernenkov, K.; Pushin, D.A. Generation and detection of spin-orbit coupled neutron beams. PNAS 2019, 116, 20328. [CrossRef]

92. Afanasev, A.V.; Karlovets, D.V.; Serbo, V.G. The Schwinger Scattering of Twisted Neutrons by Nuclei. Phys. Rev. C 2019, 100, 051601(R). [CrossRef]

93. Afanasev, A.V.; Karlovets, D.V.; Serbo, V.G. Elastic scattering of twisted neutrons by nuclei. Phys. Rev. C 2021, $103,054612$. [CrossRef]

94. Geerits, N.; Sponar, S. Twisting Neutral Particles with Electric Fields. Phys. Rev. A 2021, 103, 022205. [CrossRef]

95. Forte, M.; Heckel, B.; Ramsey, N.F.; Green, K.; Greene, G.L.; Byrne, J.; Pendlebury, J.M. First Measurement of Parity-Nonconserving Neutron-Spin Rotation: The Tin Isotopes. Phys. Rev Lett. 1980, 45, 2088. [CrossRef]

96. Michel, F.C. Parity Nonconservation in Nuclei. Phys. Rev. 1964, 133, 329. [CrossRef]

97. Karl, G.; Tadic, D. Model for scattering with parity violation. Phys. Rev. C 1977, 16, 1726. [CrossRef]

98. Petukhov, A.K.; Petrov, G.A.; Stepanov, S.I.; Nikolaev, D.V.; Zvezdkina, T.K.; Petrova, V.I.; Tyukavin, V.A. Nonconservation of spatial parity in the fission of heavy nuclei by polarized neutrons. JETP Lett. 1979, 30, 470.

99. Borovikova, N.V.; Vesna, V.A.; Egorov, A.I.; Knyaz'kov, V.A.; Kolomenskii, A.; Lobashev, V.M.; Pirozhkov, A.N.; Popeko, L.A.; Smotritskii, L.M.; Titov, N.A.; et al. Investigation of spatial parity breakdown effects in thermal polarized neutron capture reactions with the release of heavy charged particles. JETP Lett. 1979, 30, 527.

100. Petukhov, A.K.; Petrov, G.A.; Stepanov, S.I.; Nikolaev, D.V.; Zvezdkina, T.K.; Petrova, V.I.; Markova, E.S.; Ivanov, V.V.; Morozov, V.F. Asymmetry in the separation of fragments of the fission of ${ }^{235} \mathrm{U}$ and ${ }^{239} \mathrm{Pu}$ by slow polarized neutrons. JETP Lett. 1980, 32, 41.

101. Danilian, G.V. Parity violation in Nuclear Fission. Sov. Phys. Usp. 1980, 23, 3231.

102. Sushkov, O.P.; Flambaum, V.V. Parity breaking in the interaction of neutrons with heavy nuclei. Sov. Phys. Usp. 1982, $25,1$. [CrossRef]

103. Bunakov, V.E.; Gudkov, V.P. Parity violation and related effects in neutron-induced reactions. Nucl. Phys. A 1983, $401,93$. [CrossRef]

104. Alexandrovich, A.Y.; Val'skij, G.; Golosovskaya S.; Zvezdkina T.; Nikolaev D.; Petrov G.; Petrova V.; Pleva Y.; Tyukavin V. P-odd asymmetry of fragments in fission ${ }^{239} \mathrm{Pu}$ and ${ }^{235} \mathrm{U}$ by monochromatic polarized neutrons with energies $0.05-0.6 \mathrm{eV}$. Sov. J. Nucl. Phys. 1987, 45, 772 .

105. Petrov, G.A.; Valskii, G.V.; Petukhov, A.K.; Alexandrovich, A.Y.; Pleva, Y.S.; Sokolov, V.E.; Laptev, A.B.; Scherbakov, O.A. Space parity violation in nuclear fission. Nucl. Phys. A 1989, 502, 297. [CrossRef]

106. Alexandrovich, A.Y.; Gagarski, A.M.; Krasnoschekova, I.A.; Petrov, G.A.; Petrova, V.I.; Petukhov, A.K.; Pleva, Y.S.; Geltenbort, P.; Last, J.; Schreckenbach, K. New observation of space-parity violation in neutron-induced fission of ${ }^{229} \mathrm{Th},{ }^{241} \mathrm{Pu}$ and ${ }^{241} \mathrm{Am}$. Nucl. Phys. A 1994, 567, 541. [CrossRef]

107. Jesinger, P.; Danilyan, G.V.; Gagarski, A.M.; Geltenbort, P.; Goennenwein, F.; Koetzle, A.; Korobkina, Y.I.; Mutterer, M.; Nesvizhevsky, V.; Neumaier, S.R.; et al. Interference Effect in the Angular Distribution of Outgoing Particles in Ternary Fission Induced by Cold Polarized Neutrons. Phys. At. Nucl. 1999, 62, 1608.

108. Jesinger, P.; Kotzle, A.; Gagarski Gonnenwein, F.; Danilyan, G.; Pavlov, V.S.; Chvatchkin, V.B.; Mutterer, M.; Neumaier, S.R.; Petrov, G.A.; Petrov, V.O.; et al. Observation of a triple correlation in ternary fission: Is time reversal invariance violated? Nucl. Instrum. Meth. A 2000, 440, 618. [CrossRef]

109. Goennenwein, F.; Mutterer, M.; Gagarski, A.; Guseva, I.; Petrov, G.; Sokolov, V.; Zavarukhina, T.; Gusev, Y.; von Kalben, J.; Nesvizhevski, V.; et al. Rotation of the compound nucleus ${ }^{236} \mathrm{U}^{*}$ in the fission reaction ${ }^{235} \mathrm{U}(\mathrm{n}, \mathrm{f})$ induced by cold polarised neutrons. Phys. Lett. B 2007, 652, 13. [CrossRef]

110. Gagarski, A.; Gonnenwein, F.; Guseva, I.; Jesinger, P.; Kopatch, Y.; Kuzmina, T.; Leli'evre-Berna, T.; Mutterer, M.; Nesvizhevsky, V.; Petrov, G.; et al. Particular features of ternary fission induced by polarized neutrons in the major actinides ${ }^{233} \mathrm{U},{ }^{235} \mathrm{U}$ and ${ }^{239} \mathrm{P}$, ${ }^{241} \mathrm{Pu}$. Phys. Rev. C 2016 93, 054619. [CrossRef] 
111. Kopatch, Y.; Novitsky, V.; Ahmadov, G.; Gagarsky, A.; Berikov, D.; Danilyan, G.; Hutanu, V.; Klenke, J.; Masalovich, S. Measurement of the ROT effect in the neutron induced fission of ${ }^{235} \mathrm{U}$ in the $0.3 \mathrm{eV}$ resonance at a hot source of polarized neutrons. EPJ Web Conf. 2018, 169, 00010. [CrossRef]

112. Kadmensky, S.G.; Lyubashevsky, D.E.; Kostryukov, P.V. Relation of Experimental Features of P-Even, T-Odd Asymmetries in Ternary Nuclear Fission Induced by Cold Polarized Neutrons to Triple and Quintuple Scalar Correlations. Phys. Atm. Nucl. 2019 82, 267. [CrossRef]

113. Sakharov, A.D. Violation of CP Invariance, C Asymmetry, and Baryon Asymmetry of the Universe. Pisma Zh. Eksp. Teor. Fiz. 1967, 5,32 .

114. Gudkov, V.P. On the test of CP-violation models in neutron reactions. Phys. Lett. 1990, B243, 319. [CrossRef]

115. Gudkov, V.P.; Song, Y. Discovery potential in a search for time-reversal invariance violation in nuclei. Hyperfine Interact. 2013, 214, 105. [CrossRef]

116. Bowman, J.D.; Gudkov, V.P. Search for time reversal invariance violation in neutron transmission. Phys. Rev. C 2014, $90,065503$. [CrossRef]

117. Yuan, V.W.; Bowman, C.D.; Bowman, J.D.; Bush, J.E.; Delheij, P.P.J.; Frankle, C.M.; Gould, C.R.; Haase, D.G.; Knudson, J.N.; Mitchell, G.E.; et al. Parity nonconservation in polarized-neutron transmission through ${ }^{139}$ La. Phys. Rev. C 1991, $44,2187$.

118. Szymanski, J.J.; Snow, W.M.; Bowman, J.D.; Cain, B.; Crawford, B.E.; Delheij, P.P.J.; Hartman, R.D.; Haseyama, T.; Keith, C.D.; Knudson, J.N.; et al. Observation of a large parity nonconserving analyzing power in Xe. Phys. Rev. C 1996, 53, R2576. [CrossRef]

119. Skoy, V.R.; Sharapov, E.I.; Gundorin, N.A.; Popov, Y.P.; Prokofichev, Y.V.; Roberson, N.R.; Mitchell, G.E. Isotopic identification of the parity-violating neutron p-wave resonance at energy $E=3.2 \mathrm{eV}$ in Xe. Phys. Rev. C 1996, 53, R2573. [CrossRef] [PubMed]

120. Alfimenkov, V.P.; Borzakov, S.B.; Thuan, V.V.; Mareev, Y.D.; Pikelner, L.B.; Khrykin, A.S.; Sharapov, E.I. Parity Nonconservation in Neutron Resonances. Nucl. Phys. A 1983, 398, 93. [CrossRef]

121. Frankle, C.M.; Bowman, J.D.; Bush, J.E.; Delheij, P.P.J.; Gould, C.R.; Haase, D.G.; ; Knudson, J.N.; Mitchell, G.E.; Penttila, S.; Postma, H.; et al. Parity nonconservation for the 0.88-eV neutron resonance in ${ }^{81}$ Br. Phys. Rev. C 1992, 46, 1542. [CrossRef] [PubMed]

122. Shimizu, H.M.; Adachi, T.; Ishimoto, L.; Masaike, A.; Masuda, Y.; Morimoto, K. Longitudinal asymmetry and gamma-ray angular distribution in neutron radiative capture reactions. Nucl. Phys. A 1993, 552, 293. [CrossRef]

123. Gudkov, V.P. On CP violation in nuclear reactions. Phys. Rept. 1992, 212, 77. [CrossRef]

124. Beda, A.G.; Skoy, V.R. Current Status of Research on T Invariance in Neutron-Nuclear Reactions. Phys. Part. Nucl. 2007, $38,1063$. [CrossRef]

125. Okudaira, T.; Takada, S.; Hirota, K.; Kimura, A.; Kitaguchi, M.; Koga, J.; Nagamoto, K.; Nakao, T.; Okada, A.; Sakai, K.; et al. Angular distribution of $\gamma$ rays from neutron-induced compound states of ${ }^{140}$ La. Phys. Rev. C 2018, 97, 034622. [CrossRef]

126. Masuda, Y. T violation in neutron-nucleus interaction. In International Workshop on Polarized Ion Sources and Polarized Gas Jets; National Laboratory of High-Energy Physics: Ibaraki-ken, Japan, 1990; p. 249.

127. Takahashi, Y.; Yabuzaki, T.; Shimizu, H.M. Possible nuclear polarization of ${ }^{139} \mathrm{La}$ in $\mathrm{Nd} 3+: \mathrm{LaAlO}_{3}$ for the test of time reversal invariance. Nucl. Instrum. Meth. 1993, A336, 583. [CrossRef]

128. Hautle, P.; Iinuma, M. Dynamic nuclear polarization in crystals of $\mathrm{Nd} 3+: \mathrm{LaAlO}_{3}$, a polarized ${ }^{139}$ La target for a test of timereversal invariance. Nucl. Instrum. Meth. 2000, A440, 638. [CrossRef]

129. Keith, C.D. (Thomas Jefferson National Acceleration Facility, Newport News, VA, USA). Personal communication, 2019.

130. Molway, M.J.; Bales-Shaffer, L.; Ranta, K.; Basler, D.; Murphy MKidd, B.E.; Gafar, A.T.; Porter, J.; Albin, K.; Goodson, BMChekmenev, E.Y.; et al. Two-Orders-of-Magnitude Improvement in the Total Spin Angular Momentum of ${ }^{131}$ Xe Nuclei Using Spin Exchange Optical Pumping. Phys. Rev. A 2021, submitted.

131. Goodson, B.; Chekmenev, E.; Shchepin, R.; Curole, J.; Snow, W.M. Progress Toward A Search for Time Reversal Violation in Polarized Neutron Transmission on the $1.33 \mathrm{eV}$ P-Wave Resonance in Polarized ${ }^{117}$ Sn Using SABRE. Angezv. Chem. 2021, submitted.

132. Pospelov, M. Best Values for the CP-odd Meson-Nucleon Couplings from Supersymmetry. Phys. Lett. 2002, B530, 123. [CrossRef]

133. Pospelov, M.; Ritz, A. Electric dipole moments as probes of new physics. Ann. Phys. 2005, 318, 119. [CrossRef]

134. Song, Y.H.; Lazauskas, R.; Gudkov, V.P. Time reversal invariance violating and parity conserving effects in neutron-deuteron scattering. Phys. Rev. C 2011, 84, 025501. [CrossRef]

135. Mantry, S.; Pitschmann, M.; Ramsey-Musolf, M.J. Distinguishing axions from generic light scalars using electric dipole moment and fifth-force experiments. Phys. Rev. D 2014, 90, 054016. [CrossRef]

136. Fadeev, P.; Flambaum, V.V. Time-reversal invariance violation in neutron-nucleus scattering. Phys. Rev. C 2019, 100, 015504 . [CrossRef]

137. Lu, S.; Irfan, A.A.M.; Shen, J.; Kuhn, S.J.; Snow, W.M.; Baxter, D.V.; Pynn, R.; Ortiz, G. An Operator Analysis of Contextuality Witness Measurements for Multimode-Entangled Single Neutron Interferometry. Phys. Rev. A 2020, 101, 042318. [CrossRef]

138. Naudet, R. Oklo: Des Reacteurs Nucleaires Fossiles; Eyrolles: Paris, France, 1991.

139. Flambaum, V.V.; Wiringa, R.B. Enhanced effect of quark mass variation in ${ }^{229}$ Th and limits from Oklo data. Phys. Rev. C 2009, 79, 034302. [CrossRef]

140. Gould, C.R.; Sharapov, E.I. ${ }^{176} \mathrm{Lu} /{ }^{175} \mathrm{Lu}$ thermometry for the Oklo natural reactors: A new examination of old data. Phys. Rev. C 2012, 85, 024610. [CrossRef] 
141. Flambaum, V.V. Enhanced Effect of Temporal Variation of the Fine Structure Constant and the Strong Interaction in ${ }^{229}$ Th. Phys. Rev. Lett. 2006, 97, 092502. [CrossRef] [PubMed]

142. Beeks, K.; Sikorsky, T.; Schumm, T.; Thielking, J.; Okhapkin, M.V.; Peik, E. The thorium-229 low-energy isomer and the nuclear clock. Nat. Rev. Phys. 2021, 3, 238. [CrossRef]

143. Ferrero, A.; Altshcul, B.D. Limits on the time variation of the Fermi constant $G_{F}$ based on type 1A supernova observations. Phys. Rev. D 2021, 82, 123002. [CrossRef] 